\title{
Monitoring of Surface Subsidence of the Mining Area Based on SBAS
}

\author{
Yufeng $\mathrm{Zhu}^{1,2}$ \\ ${ }^{1}$ Fundamental Science on Radioactive Geology and Exploration Technology Laboratory, East China Institute of \\ Technology, NanChang, China \\ ${ }^{2}$ Central South University, Changsha, China \\ Email: yfzhu@ecit.cn \\ Xiaoli Ding ${ }^{2}$, Zhiwei $\mathrm{Li}^{2}$, Yan Luo ${ }^{1}$ \\ ${ }^{1}$ Fundamental Science on Radioactive Geology and Exploration Technology Laboratory, East China Institute of \\ Technology, NanChang, China \\ ${ }^{2}$ Central South University, Changsha, China \\ Email: lsxlding@polyu.edu.hk, zwli@mail.csu.edu.cn,yluo@ecit.cn
}

\begin{abstract}
This paper has collected 7 scenes of $L$ band PALSAR sensor radar data of a mine in FengCheng city, jiangxi province, using the Small-baseline Subset (SBAS) method to invert the surface subsidence of the mine. Baselines of interference less than $800 \mathrm{~m}$ has been chosen to constitute short baseline differential interference atlas, using pixels whose average coherent coefficient was larger than or equal to 0.3 as like high coherent point target, using singular value decomposition (SVD) method to calculate deformation phase sequence based on these high coherent points, and the accumulation of settlements of study area of different period had been obtained, so as to reflect the ground surface settlement evolution of the settlement of the area. The results of the study has showed that: SBAS technology has overcome coherent problem of the traditionality D-InSAR technique, continuous deformation field of surface mining in time dimension of time could been obtained, characteristics of ground surface settlement of mining subsidence in different period has been displayed, so to improve the accuracy and reliability of the monitoring results.
\end{abstract}

Index Terms-D-InSAR, Small-baseline Subset, Mining subsidence, deformation monitoring

\section{INTRODUCTION}

Ground settlement and subsidence caused by mining is a destruction disasters often happening in mining area, and it is one of the most important geological disasters in China. Ground subsidence of mined-out area not only destroys all kinds of architectural, engineering, water conservancy, transportation facilities and farmland, it is also a threat to people's life and property safety, and it has become an important factor which influences and restricts the sustainable development of mining cities[1,2]. Therefore, it is necessary to use the advanced technology to monitor and control damage caused by the ground surface settlement, which can guarantee the sustainable development of mining area.

Differential Interferometric Synthetic Aperture echoes(D-InSAR) is a surface deformation monitoring method recently developed, monitoring the coal mine area of all-weather, non-contact, planar, fast and accurate, with centimeters or $\mathrm{mm}$ level. However D-InSAR technology focuses on the single deformation within short time interval, so SAR image which can be used is little, without redundant observations. When the deformation phase is not far from phase noise, deformation phase can't be separated effectively, and reliability of deformation calculation is poor [3]. Because of incoherent of the time and the baselines, orbit error and the influence of the atmosphere, D-InSAR single discrete settlements cannot be connected in most cases, and it is difficult to detect the deformation sequence, and the development and evolution of settlement of the study area coal mine can't be obtained.

According to the D-InSAR technology limitations, in recently years, some scholars abroad calculated the deformation rate effectively by using least squares estimation method in the high coherence point target, through virtual observation method, and in which accuracy and reliability of D-InSAR technology deformation calculation has been improved [4,5]. Ferretti, etc. (2000) first proposed Permanent Scatter technology, choosing a series of phase stable point as PS point according to a certain criterion in a group of radar interference image given, and after removing DEM error, atmospheric delay phase, orbit error and other noise of PS points according to the given phase model, then the exact deformation of surface shape can be obtained [6,7].

Berardino [8] (2002) and Lanari [9] (2004) proposed SBAS method, using small baselines combination to get interference measurement, calculating of the combination of much small baselines using SVD method can effectively to solve discontinuous time problems caused by space between baseline of different SAR data which is too long to improve resolution of monitoring time, so that accuracy of D-InSAR technology deformation calculation has been improved further. 
In this paper, a coal mine area in FengCheng city, Jiangxi province has been selected as the research area, the L-band PALSAR data of the region has been chosen to do time series analysis using SBAS technology, and time series deformation field could be obtained, and mining subsidence funnel could be detected and positioned. Development situation of mining subsidence funnel has been analyzed, to reveal the rule of evolution of subsidence funnel according with time.

\section{SMall-BASEline SubSet Theory}

In 2002, Berardion and others proposed that SBAS method was used to study low resolution and large scale of deformation in order to get deformation time sequence diagram [8]. According to the principle of SBAS technology — - baseline distance sets in SAR image is small, baseline distance between the set of SAR image is big, the SAR data acquired would be combined into lots of combinations, and then baselines combinations could be solved together by using SVD method or Least squares (LS, Least Square) method. This method can effectively weak the influence incoherent of the space, and at the same time, overcome DEM error and inconsistent of atmospheric phase, so that deformation diagram got could be more continuous in space. This specific principle can be following as three steps:

\section{A. Least Squares (LS)}

Suppose that there are $N+1$ scenes covering the same area of SAR image, obtaining in time $t_{0}, t_{1}, \cdots, t_{N}$, and every image can interfere with another image at least, this means that each short baseline could be combined by at least two images. Based on the above assumptions, $\mathrm{M}$ numbers of differential interferograms could be obtained, in which $\mathrm{M}$ meets the following inequality ( $\mathrm{N}$ is assumed to odd):

$$
\frac{N+1}{2} \leq M \leq N\left(\frac{N+1}{2}\right)
$$

Suppose that the $\mathrm{j}$ scene interferogram could be produced by SAR image obtained from $t_{A}$ and $t_{B}$. Removing topographic phase, and assuming $t_{B}>t_{A}$, then in bearing - distance pixel coordinate system $(x, r)$, the interference phase in $(x, r)$ of the interference figure can be expressed as:

$$
\begin{aligned}
\delta \phi_{j}(x, r) & =\phi\left(t_{B}, x, r\right)-\phi\left(t_{A}, x, r\right) \\
& \approx \frac{4 \pi}{\lambda}\left[d\left(t_{B}, x, r\right)-d\left(t_{A}, x, r\right)\right]
\end{aligned}
$$

In formula (2), $\lambda$ is a radar center wavelength, $d\left(t_{B}, x, r\right)$ and $d\left(t_{A}, x, r\right)$ are accumulated form variables of LOS respectively to the reference time $t_{0}$, thus $d\left(t_{0}, x, r\right) \equiv 0$; Of course $d\left(t_{i}, x, r\right)(\mathrm{I}=1, \ldots, \mathrm{N})$ is used to respect the deformation time series obtained, and the corresponding phase was set up as $\phi\left(t_{i}, x, r\right)$, and then $\phi\left(t_{i}, x, r\right) \approx 4 \pi d\left(t_{i}, x, r\right) / \lambda$. It should be pointed out that, in the formula (2) atmospheric phase, residual topographic phase and incoherent phenomenon are not be considered in phase $\phi\left(t_{i}, x, r\right)$; And assume that all phase signals are unwrapping, and a pixel with a type variable known and be corrected is used as starting point unwrapped.

Vector of $\mathrm{N}$ number of the unknown phase value corresponded with the pixel shape variables analyzed could be expressed as:

$$
\phi^{T}=\left[\phi\left(t_{1}\right), \ldots, \phi\left(t_{N}\right)\right]
$$

Vector consists from $\mathrm{M}$ phase unwrapping from the differential interference graphical could be expressed as:

$$
\delta \phi^{T}=\left[\delta \phi_{1}, \ldots, \delta \phi_{M}\right]
$$

The corresponding time series of IE image and IS image could be expressed as respectively:

$$
I E=\left[I E_{1}, \ldots, I E_{M}\right] \quad I S=\left[I S_{1}, \ldots, I S_{M}\right]
$$

Suppose that IE image and IS image could be arranged according to time, just $I E_{j}>I S_{j}$, and $\mathrm{j}=1, \cdots, \mathrm{M}$, then the phase of $\mathrm{j}$ scene of interferogram could be expressed as:

$$
\delta \phi_{j}=\phi\left(t_{I E_{j}}\right)-\phi\left(t_{I S_{j}}\right), \mathrm{j}=1, \cdots, \mathrm{M}
$$

For all interferogram, linear model in formula (6) could be expressed as $\mathrm{M}$ number equations of $\mathrm{N}$ unknown values, the matrix form is:

$$
A \phi=\delta \phi
$$

Among them A is $M \times N$ matrix, each row corresponds to an interferogram, each column corresponds to a SAR image of one time, the column of IE image is +1 , the column of IS image is -1 , the rest is zero. If $\mathrm{M} \geqslant \mathrm{N}$ and the rank of $\mathrm{A}$ is $\mathrm{N}$, then do it according to the least square method:

$$
\phi=A^{\#} \cdot \delta \phi, \quad A^{\#}=\left(A^{T} A\right)^{-1} A^{T}
$$

\section{B. Singular Value Decomposition (SVD)}

When $\mathrm{M}<\mathrm{N}$, matrix $\mathrm{A}$ is rank-defect, $A^{T} A$ is a singular matrix; suppose that there are $L$ number of different baseline sets, the rank of matrix is $\mathrm{N}-\mathrm{L}+1$, and the equations will have an infinite number of solutions. In order to solve this problem, it needs to use the singular value decomposition of matrix method, just doing SVD of the coefficient matrix A, and the minimum norm of the sense of least squares could be obtained. The concrete implementation steps are as follows:

$$
A=U S V^{T}
$$

In formula, $\mathrm{U}$ is orthogonal matrix $M \times M$ dimensional, composed by the characteristic vector $U_{i}$ of $A A^{T} ; \mathrm{V}$ is orthogonal matrix $N \times M$ dimensional, composing by the characteristic vector $v_{i}$ of $A A^{T} ; \mathrm{S}$ is diagonal matrix $M \times M$ dimensional, and diagonal element pixel is characteristic value $\sigma_{i}$ of $A A^{T}$. Assume that rank of $\mathrm{A}$ is $\mathrm{R}$, the former $\mathrm{R}$ characteristic value of $A A^{T}$ is nonzero value, then $M-R$ characteristic value is 0 . A pseudo inverse matrix is defined as $A^{+}$, then for valuation, the value of $\hat{\phi}$ acquired by squares constraints can be expressed as follows:

$$
\hat{\phi}=A^{+} \delta \phi, A^{+}=V S^{+} U^{T}
$$

So least squares phase estimate value in minimum norm could be obtained as:

$$
\hat{\phi}=\sum_{i=1}^{R} \frac{\delta \phi^{T}}{\sqrt{\sigma_{i}}} v_{i} u_{i}
$$




\section{Extraction of Linear Deformation Rate}

In order to obtain the physical meaning of the sedimentation sequence, phase is used to respect the product of average phase velocity and time in formula (9), namely:

$$
v^{T}=\left[v_{1}=\frac{\phi_{1}}{t_{1}-t_{0}}, \ldots, v_{N}=\frac{\phi_{N}-\phi_{N-1}}{t_{N}-t_{N-1}}\right]
$$

Instead the phase of formula (6),

$$
\sum_{k=I S_{J}+1}^{I E_{J}}\left(t_{k}-t_{k-1}\right) v_{k}=\delta \phi_{j}, j=1, \cdots, \mathrm{M}
$$

So a new matrix equation could be obtained, namely:

$$
B v=\delta \phi
$$

In formula (13), $\mathrm{B}$ is a $M \times N$ matrix. For the first $\mathrm{j}$ line, columns located in acquisition time of IE and IS images, $B(j, k)=t_{k}-t_{k-1}$, and the other $B(j, k)=0$, the average deformation rate phase value $v_{k}(\mathrm{k}=1, \cdots, \mathrm{N})$ of each time interval of $\mathrm{N}+1$ number images could be worked out by the least squares method or SVD decomposition, and cumulative surface linear deformation phase acquisition time from the first image to the other image could be calculated by multiplication accumulation of $v_{k}$ and interval times.

After acquisition of linear deformation phase, a new linear model can be established by other linear phase contribution parameters (error and track error caused by DEM), so that deformation valuations inversion could be obtained more accurately. In addition, based on the linear model, the nonlinear deformation phase and atmospheric phase could be separated by filter according to the different characteristics of the residual phase in time and space performance $[6,7]$.

\section{EXPERIMENTAL RESEARCH}

In order to verify the validity and practicability of the technology in surface subsidence monitoring, a coal mine area in FengCheng city, Jiangxi province has been chosen as the research object, using SBAS technology for time series analysis, sedimentation sequence diagram from June 2007 to May 2008 could be obtained.

\section{A. The Experimental Datas}

In this paper, 7 scenes the 1-band SAR data of PALSAR ALOS satellite sensors covered plateau lake coal mine area and a series of disposal of no "empty" global SRTM V4 DEM data with 3 seconds accuracy (resolution for $90 \mathrm{~m}$ ) which nominal absolute elevation accuracy is $\pm 16 \mathrm{~m}$ and nominal absolute plane precision is $\pm 20 \mathrm{~m}$ provided by spatial information association (CSI) of CGIAR were used. The specific parameters of image information are shown in table 1 . In the table image of FBD mode should be 2 times over sampling in the distance in order to keep consistent resolution with FBS mode. In removing topographic phase information with collecting DEM, it should do 6 times over sampling of DEM.
TABLE1

THE SCHEDULE OF SAR DATA

\begin{tabular}{|l|l|l|l|l|}
\hline $\begin{array}{l}\text { Image } \\
\text { Number }\end{array}$ & $\begin{array}{l}\text { Date } \\
\text { (YYYYMMDD) }\end{array}$ & $\begin{array}{l}\text { Track } \\
\text { Number }\end{array}$ & $\begin{array}{l}\text { Observation } \\
\text { Mode }\end{array}$ & $\begin{array}{l}\text { Polarization } \\
\text { Mode }\end{array}$ \\
\hline 1 & 20070614 & 07389 & FBD & HH+HV \\
\hline 2 & 20070730 & 08060 & FBD & HH+HV \\
\hline 3 & 20070914 & 08731 & FBD & HH+HV \\
\hline 4 & 20071030 & 09402 & FBD & HH+HV \\
\hline 5 & 20071215 & 10073 & FBS & HH \\
\hline 6 & 20080130 & 10744 & FBS & HH \\
\hline 7 & 20080501 & 12086 & FBD & HH+HV \\
\hline
\end{tabular}

For the 1-band of ALOS satellite, less than or equal to $800 \mathrm{~m}$ of the baseline can be used as a small baseline, the space of incoherent could be improved, and the coherence increased obviously [3]. Similarly, L band PALSAR data were selected in this experiment, baseline less than $800 \mathrm{~m}$ were selected to produce 11 small baselines interfere pairs, and with the use of SBAS method, vertical baseline set distribution could be seen as figure 1 .

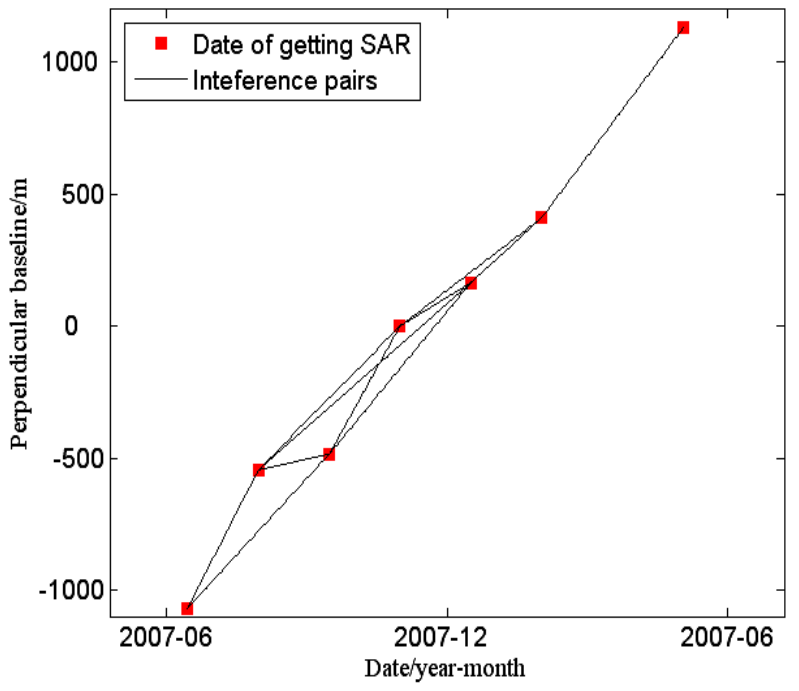

Figure 1. Vertical baseline set pattern

\section{B. Data Processing}

Using SBAS method to process data, firstly SAR image data acquired were done with differential interference preproccess of D-InSAR method, and time series D-InSAR analysis model were established to calculate settlement rate and cumulative type variable of each time based on selected high coherent points. Data processing flow of SBAS method was shown in figure 2 .

\section{1) Pretreatment}

Before the D-InSAR time series analysis, small base set interferogram sequence was generated by SAR data "two rail" method differential interference processing in GAMMA software of Swiss GAMMA Company in this paper.

(1) Selection of IE image

Because SBAS method is used to analyze the time series of identical point, as PS technology, interferograms 
should be registered to the same coordinate system in this method. Therefore, a picture of IE image should be chosen, and other images should be registered to the IE image, and then single visual interference figure could be generated based on the same main image. The image of the vertical baseline less than $800 \mathrm{~m}$ of interference and the time base, vertical baseline and doppler centroid frequency baseline of minimizing was chosen as IE image. IE image selected in this paper was SAR image obtained in October 30, 2007. Three baseline statistical results could be seen as table 2 .

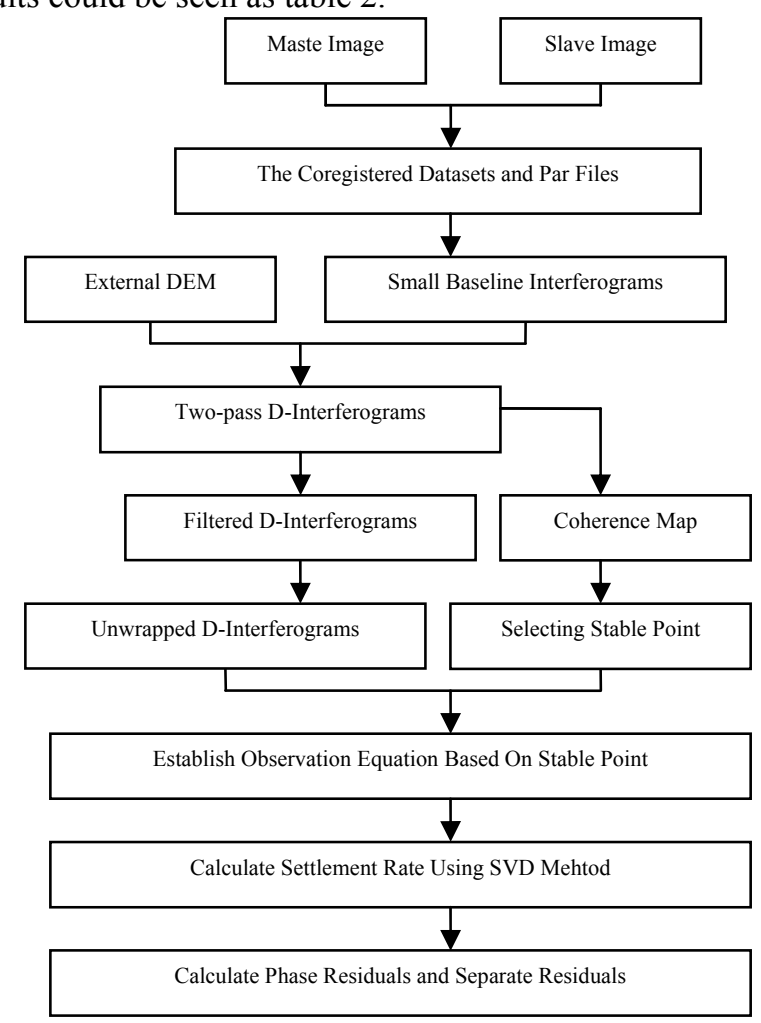

Figure 2. The flowchart of data processing in SBAS

TABLE2

THE STATISTICAL TABLE ABOUT THREE BASELINES

\begin{tabular}{|l|l|l|l|l|}
\hline $\begin{array}{l}\text { Image } \\
\text { Number }\end{array}$ & $\begin{array}{l}\text { Sum of } \\
\text { Time }\end{array}$ & $\begin{array}{l}\text { Sum of } \\
\text { Vertical } \\
\text { Baseline }\end{array}$ & $\begin{array}{l}\text { Sum of } \\
\text { Doppler } \\
\text { frequency }\end{array}$ & $\begin{array}{l}\text { Sum of } \\
\text { Three } \\
\text { Baselines }\end{array}$ \\
\hline 20070614 & 1012 & 7085.1524 & 45.3596 & 8142.5120 \\
\hline 20070730 & 782 & 4467.0160 & 44.5616 & 5293.5776 \\
\hline 20070914 & 644 & 4288.3927 & 74.4414 & 5006.8341 \\
\hline 20071030 & 598 & 3803.8635 & 51.6112 & 4453.4747 \\
\hline 20071225 & 644 & 3967.3606 & 86.5655 & 4697.9261 \\
\hline 20080130 & 782 & 4701.1704 & 66.9226 & 5550.0930 \\
\hline 20080501 & 1242 & 8304.1775 & 44.1424 & 9590.3199 \\
\hline
\end{tabular}

For the 1-band of ALOS satellite, less than or equal to $800 \mathrm{~m}$ of the baseline can be used as a small baseline, the space of incoherent could be improved, and the coherence increased obviously [3]. Similarly, L band PALSAR data were selected in this experiment, baseline less than $800 \mathrm{~m}$ were selected to produce 11 small baselines interfere pairs, and with the use of SBAS method, vertical baseline set distribution could be seen as figure 1 .

(2) Generation of differential interference pattern

Interferogram was generated by SLC differential interference in GAMMA commercial software Swiss GAMMA Company. This process was the foundation of D - InSAR time series analysis. Specific operation processment was as follows:

IE image should be cut, the scope should include the study area and have obvious relief change, and it is an advantage to the registration of study area of SAR intensity map simulated by DEM and IE image intensity map. In this paper the size of the cut was 7000 pixel in the distance, 17500 pixels in the bearing;

The other image was registered to IE image, and single visual interferogram was generated based on the same IE image;

The small base set interference was registered another, generating more apparent small baseline interference atlas of 5 bearing ,2 distance (image size: 3500 pixel * 3500 pixel); Then the small baseline interference atlas was interfered using two rail differential interference and filtering, and then differential interference atlas could be obtained after filter; Further utilizing the MCF method for unwrapping differential interference graph, then unwrapping differential interference atlas under radar coordinates could be obtained;

Due to orbit data of PALSAR radar image was not precise, similar "the ground effect" of the interference fringes (track error phase) was concluded in the filtered differential interference. Unwrapping differential interference figure of orbit error phase removed could be obtained by difference interferogram unwrapping minus orbit error phrase, using the least squares fitting method in this paper. The least squares fitting method of orbit error removed was as follows:

From the filtered difference interferogram, it could be found that orbit error was in obvious linear trend distribution, in radar system, the linear trend was shown with type double linear model $[11,12]$ :

$$
\phi_{\text {orbit_error }}=a_{0}+a_{1} \cdot x+a_{2} \cdot y+a_{3} \cdot x \cdot y
$$

In formula, $\phi_{\text {oribt error }}$ is track error phase; $\mathrm{X}$ is the distance coordinate of radar coordinate system, $y$ is azimuth coordinates of radar coordinates system( $\mathrm{x}$ and $\mathrm{y}$ are pixel position of differential interference image); $a_{0} 、 a_{1} 、 a_{2}$ and $a_{3}$ are undetermined coefficient of model, which can obtain through the least squares method. Formula of matrix form was:

$$
\phi=M \cdot A
$$

$$
\text { Among them } \quad \phi=\left[\begin{array}{llll}
\phi_{1} & \phi_{2} & \cdots & \phi_{\mathrm{n}}
\end{array}\right]
$$$$
A=\left[\begin{array}{llll}
a_{0} & a_{1} & a_{2} & a_{3}
\end{array}\right] \text {; }
$$

$$
M=\left[\begin{array}{cccc}
1 & x_{1} & y_{1} & x_{1} \cdot y_{1} \\
1 & x_{2} & y_{2} & x_{2} \cdot y_{2} \\
\vdots & \vdots & \vdots & \vdots \\
1 & x_{\mathrm{n}} & y_{n} & x_{n} \cdot y_{n}
\end{array}\right]
$$


The least squares solution was:

$$
A=\left(M^{\prime} M\right)^{-1} M^{\prime} \phi
$$

The specific processment of Least squares fitting method to remove orbit error phase was:

Step 1: some points were selected evenly in the differential interferogram of filtering and unwrapping, in order to improve the accuracy of estimation, it should be ensured that the chosen points were located in a relatively stable area and they were of high coherence.

Step 2: The formula model (16) was established, and the undetermined coefficients $a_{0}, a_{1}, a_{2}$ and $a_{3}$ could be calculated through the selected points in step 1 by the least squares.

Step 3: the undetermined coefficient calculated were substituted into formula 14 to get the orbit error phase of the whole image. Then removing the orbit error phase from the differential interference figure unwrapping, differential interference figure unwrapping could be obtained.

Differential interference atlas unwrapping in the geographic coordinates could be obtained by geocoding of the differential interference graph unwrapping which has removed the orbit error.

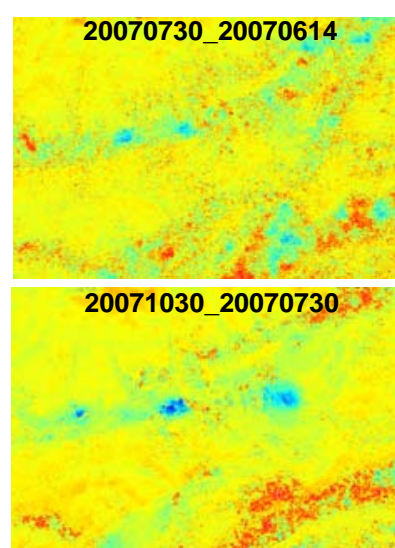

20071215_20070914
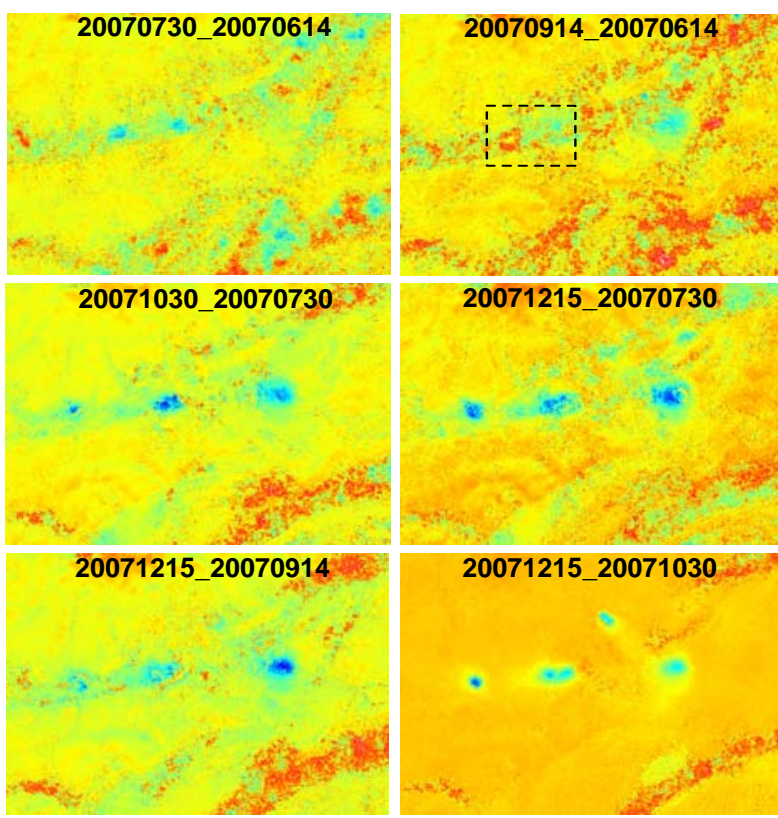

$20071215 \_20071030$
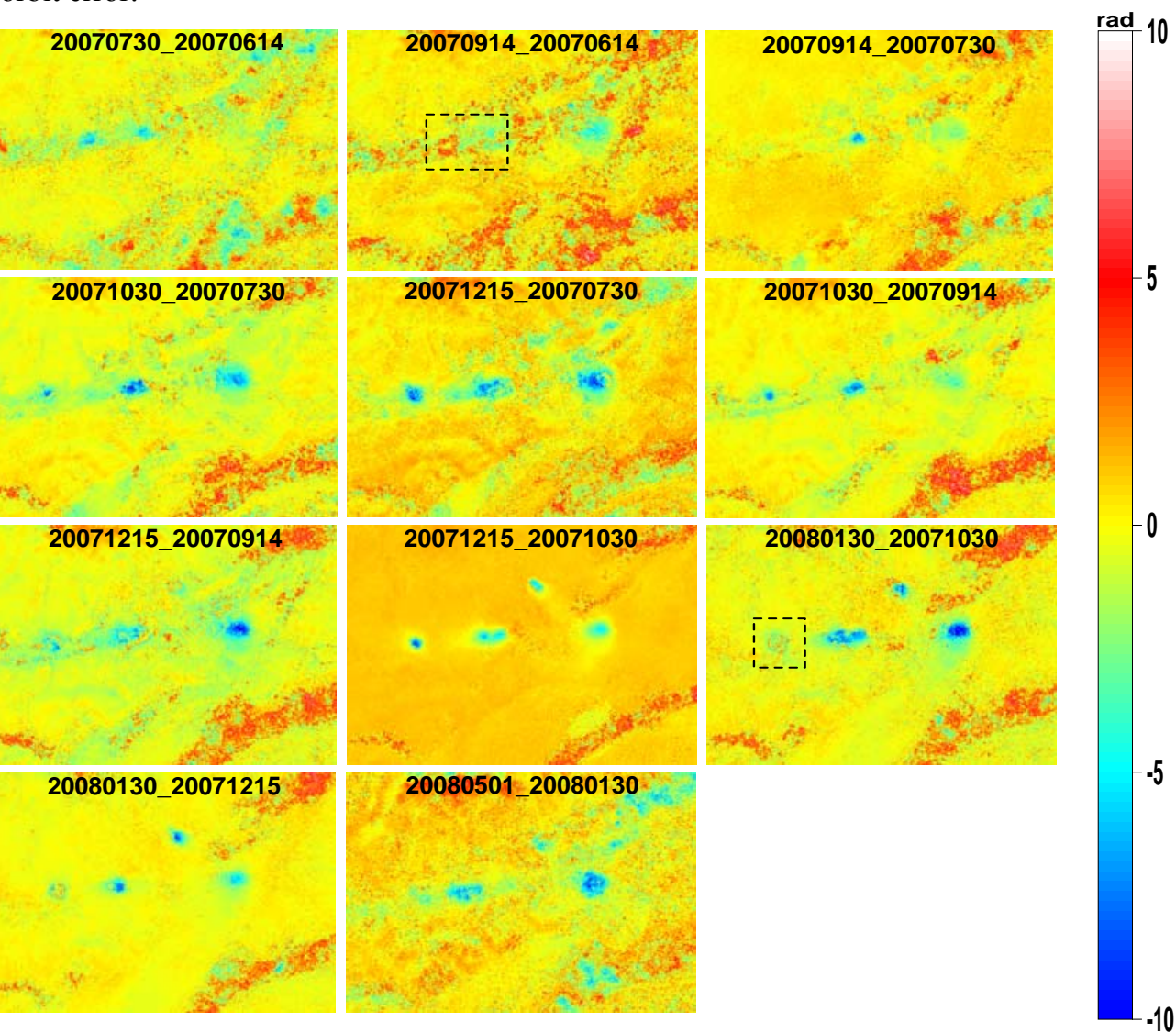

Figure 3. The unwrapped interferograms in MCF

\section{2) Choice of high coherent targets}

Before calculating deformation rate and DEM error by use of SBAS time series analysis model, the first was to choose the high coherent target. The chosen of the high
Due to the small mining area, in order to reflect the study area of the surface settlement clearly and reduce the workload, data processing using only a small image map

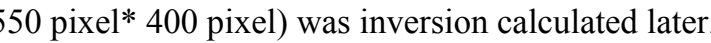

The unwrapping phase atlas of small range geographic 20070614, and it was due to bigger baseline of the interference. And in the same the field was in summer, the surface vegetation was , which was leading to the poor coherence of dotted area from the interference of $20080130 \_20071030$ is interference was in winter, plant was rare, which ould not be affected by time incoherent, but during 15 2007 to 2008 on January 30, the study area come errors in unwrapping results. 
high coherent target are amplitude threshold value method and correlation coefficient threshold value method. From the experimental, density of high coherent point by the coherent coefficient threshold value method was bigger than density of high coherent point by the amplitude threshold value method [12]. Amplitude threshold value method is used to estimate phase stability using pixel strength stability instead of coherence, so this often needs more SAR image (generally not less than 30 picture), and the statistical characteristic of the amplitude can be estimated correct. And in time series, stable high coherent point could be found by analyzing the strength of the pixel, which involves complicated mathematics method, and need do a large number of simulation experiments to determine the threshold value. Due to the small amount of data in this paper was small, simple algorithm coherent coefficient threshold value method was chosen to selected high coherent point. Selection criteria: coherent map of time series was taken average, the threshold value was chosen by average coherent coefficient of the cumulative images. Namely:

$$
\gamma_{\text {mean }}=\frac{1}{M} \sum_{j-1}^{M} \gamma_{j} \geq \gamma^{T}
$$

In formula (17), $\gamma_{j}$ was coherent coefficient of coherent map of the first $j$ amplitude difference interferogram corresponding; $\lambda^{T}$ was coherent coefficient threshold value. In the determination of the correlation coefficient threshold value, it should not only consider the number of the pixel, but also consider the quality of pixel [13]. Considering actual coherence of the study area, if the choice of threshold value is large, it would lose the advantages of large area coverage, so in this paper 0.3 was taken as a threshold value, the average coherent value which was greater than and equal to 0.3 point would be chosen as high coherent target. The study area average coherent diagram was as shown in figure 4 .

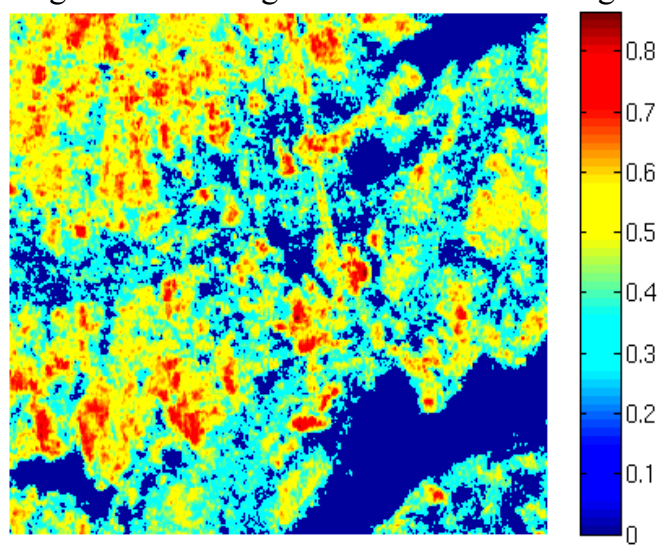

Figure 4. Mean coherence map of study region

\section{3) SBAS processing}

In the selection of high coherent target, a linear deformation rate of the linear model and constructs the system of equations is adopted in this paper, the method of singular value decomposition (SVD) to solution equations, it is estimated that interval linear settlement rate each time. Each time settlement rate in time domain integral can get on the accumulation of each time form variables

\section{Results Analysing}

Differential interference atlas unwrapping of study region after geocoding has been analyzed by SBAS method, and each time of settling diagram of mining area surface could be obtained, as shown in figure 5 .

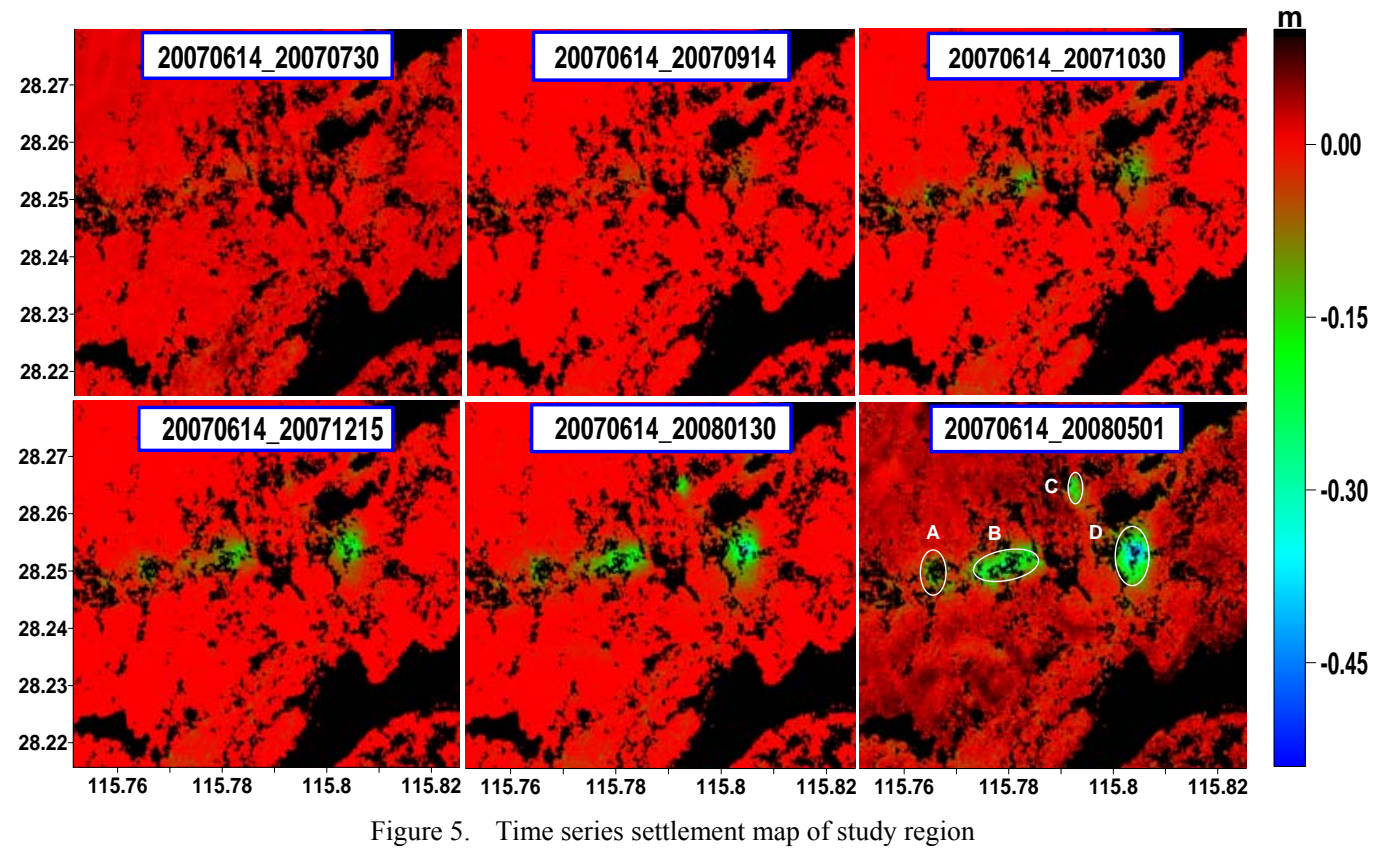

From figure 5 it could be seen that the settlement and settlement area of mining area surface has been growing, forming subsidence funnel $\mathrm{A}, \mathrm{B}, \mathrm{C}$ and $\mathrm{D}$ during this period of time. The accumulated settlement and subsidence area of subsidence funnel $\mathrm{A}$ and $\mathrm{C}$ were small in this period of time, because of slow settlement in early 
production period; Accumulated settlement of subsidence funnel $\mathrm{B}$ and $\mathrm{C}$ were bigger in this period of time, because of sedimentation acceleration in mining active phase. In order to analyze the evolution of subsidence funnel and subsidence area, the accumulation of subsidence area in different period should be statistical analyzed, and the results of statistical could be seen in table 2 . It could be clearly seen in table 2 that subsidence area of the study area was gradually expanding as times goes on. Due to the influence of ice disaster the SAR image of January 30, 2008, overall coherence of this group of image combination of the differential interferogram was very poor, leading to a certain deviation interfere with the unwrapping results, so there were unsmooth shown in subsidence area of January 2008,30 .

TABLE III.

ACCUMULATED SUBSIDENCE AREA

\begin{tabular}{|l|l|l|l|l|l|}
\hline \multirow{2}{*}{ Date } & \multicolumn{4}{|l}{ Subsidence area (Unit: $\mathrm{m}^{2}$ ) } \\
\cline { 2 - 6 } & $\begin{array}{l}\text { Settle } \\
\text { ment }>0.1 \mathrm{~m}\end{array}$ & $\begin{array}{l}\text { Settle } \\
\text { ment }>0.2 \mathrm{~m}\end{array}$ & $\begin{array}{l}\text { Settle } \\
\text { ment }>0.3 \mathrm{~m}\end{array}$ & $\begin{array}{l}\text { Settle } \\
\text { ment }>0.4 \mathrm{~m}\end{array}$ & $\begin{array}{l}\text { Settle } \\
\text { ment }>0.5 \mathrm{~m}\end{array}$ \\
\hline 20070730 & 591.0588 & 0 & 0 & 0 & 0 \\
\hline 20070914 & 1418.5000 & 0 & 0 & 0 & 0 \\
\hline 20071030 & 104970 & 0 & 0 & 0 & 0 \\
\hline 20071215 & 219050 & 9693.4000 & 0 & 0 & 0 \\
\hline 20080130 & 408890 & 7565.6000 & 7683.8000 & 0 & 0 \\
\hline 20080501 & 762580 & 190680 & 59106 & 12058 & 470.8470 \\
\hline
\end{tabular}

In order to analyze the development of subsidence funnel further, subsidence funnel $\mathrm{A}, \mathrm{C}$ and $\mathrm{D}$ has been selected to do profile analysis, in order to observe their time series of the changes of the settlement. The profile of settlement according to time series of center section of funnel $\mathrm{D}$ in the column and row direction has been shown in figure 6. From the figure it could been clearly reflected that the settlement of the subsidence funnel gradually increase as time increases, so it could be concluded that it was doing the coal mining in this work face during this period of time.

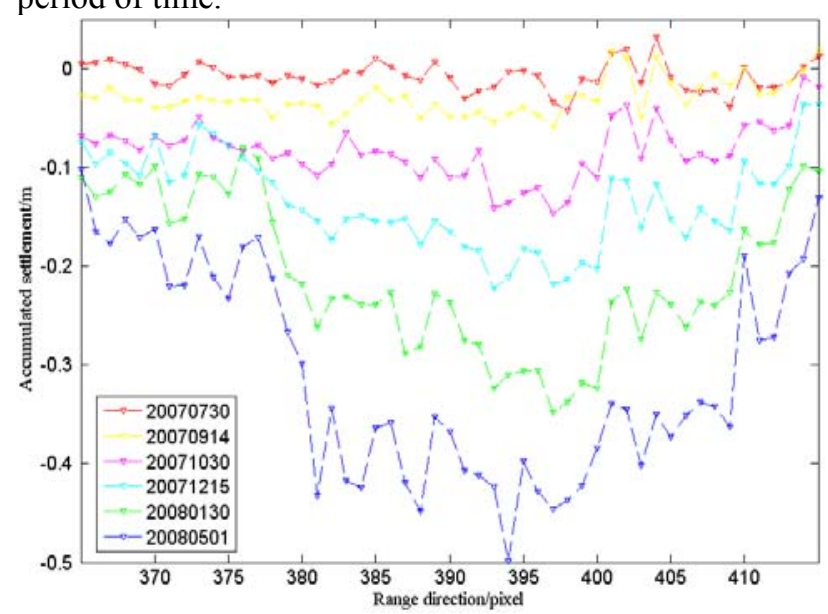

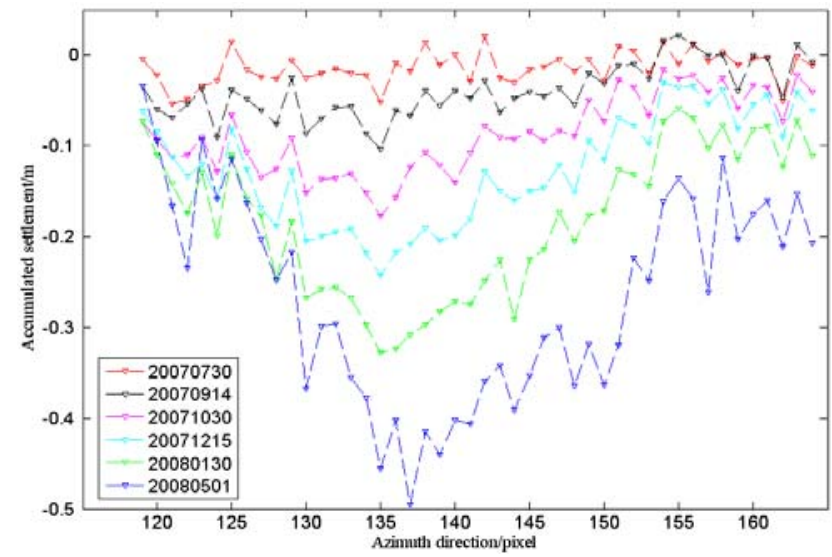

Figure 6. Time series settlement profiles of subsidence funnel D in distance and azimuth direction

Also funnel $\mathrm{A}$ and $\mathrm{C}$ area has been analyzed respectively, as shown in figure 7 and figure 8 . The settlement of funnel $\mathrm{C}$ and funnel $\mathrm{D}$ area were continuous subsiding as times goes by, as funnel B area. But there was discontinuity of settlement in the space in the figure 7 and 8 , due to the poor coherence caused by near to water and crops.

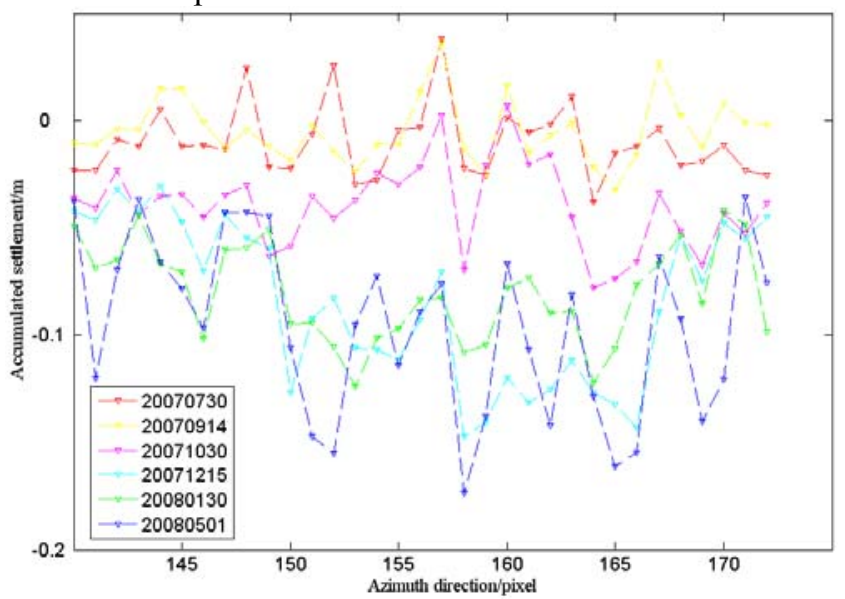

Figure 7. Time series settlement profile of subsidence funnel A in bearing direction

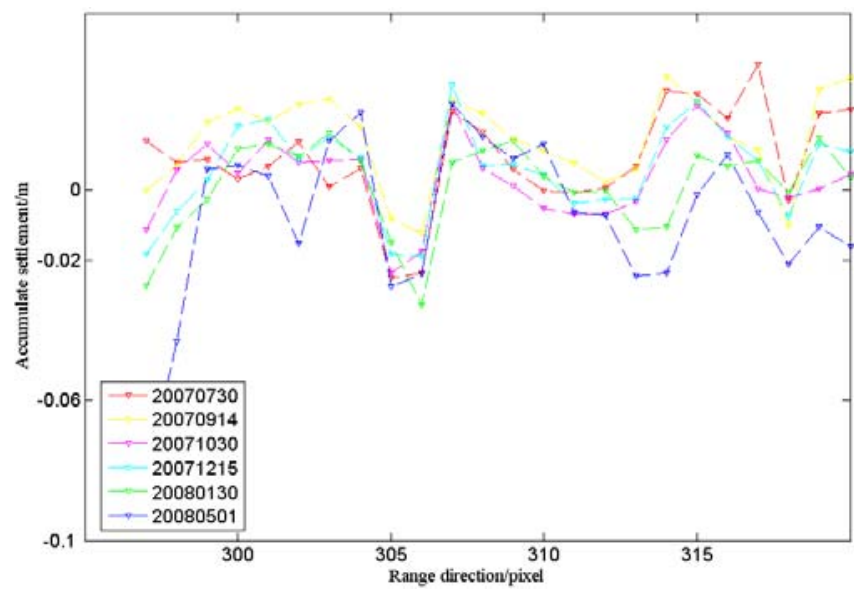

Figure 8. Time series settlement profile of subsidence funnel $\mathrm{C}$ in distance direction 


\section{CONCLUTION}

In this paper SBAS method has been tested with 1band PALSAR sensor radar data, and the experiment results showed that this method can effectively get the subsidence sequence diagram of coal mine area surface. Although SBAS technology has improved D-InSAR technology results well, the quantity of using data was limited, leading to a certain error in results. As the data have two scene data influenced by ice disaster, the overall coherence was poorer, and there were some deviation in unwrapping results, and the accuracy of inversion deformation has been affected. Due to the smaller mining area, and compared to the atmospheric error phase of space low frequency characteristics, settlement has higher spatial frequency, the differential interferometric phase was led by the phase signal of settlement, so in this paper the influence of atmospheric error hasn't been considered[14]. In addition, the phase error caused by inaccuracy DEM has not been considered in the data processing, and it is the further step to do research on how will DEM error can be used as a parameter to estimate and separate effectively.

\section{ACKNOWLEDGMENTS}

This paper is supported by Natural Science Foundation of China (40874010), Natural Science Foundation of Jiangxi China (2012ZBAB216001), State Key Laboratory Breeding Base of Nuclear Resources and Environment, East China Institute of Technology (101115).

\section{REFERENCES}

[1] Y.F. Zhu, X.L. Ding, Z.W. Li, S.J. Zhou, "Discussion on the Application of GPS Using in Marine Construction Survey," Journal of Computers. Finland, Vol.7, pp. 16631670, 2012.

[2] Y.F. Zhu, X.L. Ding, Y. Luo, Z.W. Li, "Analysis on the Application of GPS-RTK Technology using in Yangkou

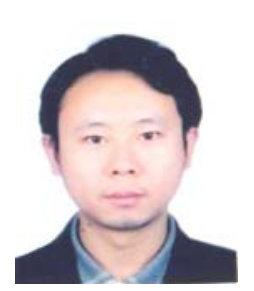

Yufeng Zhu was born in Jinxian, Jiangxi, China, in 1981. He received the Master degree in cartography and geographic information engineering from East China Institute of Technology (ECIT), Fuzhou, China, in 2005. He is currently studying toward the Doctor degree in geodesy and surveying engineering, School of Geosciences and Infor-physics, Central South University (CSU), Changsha, China.

As a teacher of East China Institute of Technology, Fuzhou, China, his research topics include intelligence algorithm, research of GIS, mining surveying, and application of InSAR technology. man-made Island in Nantong City," Journal of Networks. Finland, Vol.6, pp. 1444-1451, 2011.

[3] H.J. Yin, J.J. Zhu, Z.W. Li, X.L. Ding, C.C. Wang, "Ground Subsidence Monitoring in Mining Area Using DInSAR SBAS Algorithm," Acta Geodaeticaet Cartographica Sinica. China, Vol.40, pp.52-58, 2011.

[4] Usai S,KLEES R, "SAR Interfermetry on a Very long Time Scale:a study of the Interferometric Characteristics of Man made Features," IEEE Transactions on Geoscience and Remote Sensing.U.S, Vol.37, pp.2118-2123. 1999.

[5] Usai S, "A Least Squares Database Aproach for SAR Interfermetry Data," IEEE Transactions on Geoscience and Remote Sensing. U.S, Vol.41, pp.753-760, 2003.

[6] Ferretti A,Prati C,and Rocca F, "Nonlinear Subsidence Rate Estimation Using Permanet Scatters in Differential SAR Interferometry," IEEE Transactions on Geoscience and Remote Sensing. U.S, Vol.38, pp.2202-2212, 2000.

[7] Ferretti A,Prati C,and Rocca F, "Permanent Scatters in SAR interferometry," IEEE Transactions on Geoscience and Remote Sensing. U.S, Vol.39, pp.8-20, 2001.

[8] Berandino P,Fornaro G,Lanari R.A new algorithm for surface deformation monitoring based on small baseline differential interferograms[J].IEEE Transactions on Geoscience and Remote Sensing,2002,40(11):2375-2383.

[9] Lanri R,Mora O,Manunta M, "A small_baseline approach for investigating deformation on full_resolution differential SAR interferograms," IEEE Transations on Geoscience and Remote Sensing. U.S, Vol.42, pp.1377-1386, 2004

[10] H.Zhang, C.Wang, T.Wu, Y.X.Tang, Research on DInSAR method Based on the coherent target. Science publications, China 2009.

[11] H.L.Yang, J.H. Peng, D.X. Zhang, S.H. Li, "Infuluence of orbital errosr on InSAR data processing," Journal of Geomatics Science and Technology. China, Vol.29, pp.118-121, 2012.

[12] X.Y. Dong, Research on earthquake deformation monitoring Based on image matching technology, Central South University, China 2011.

[13] X.F.He, M.He, Earth observation data processing method and comprehensive measurement based on InSAR Technology. Science publications, China 2012.

[14] B.W.Huang, X.G.Song, Z.J.Wang, X.J.San, "Research on ground subsidence monitoring of Ge Ting coal mine based on D-InSAR and GIS techniques," Geotechnical Investigation \& Surveying. China, Vol.4, pp.55-60, 2012. 